\title{
Effects of training with nintendo wii on balance and mobility in institutionalized elderly
}

\author{
Lara Alves Moreira,2, Lívia Marcondes Faber'1, Marcos Eduardo Scheicher ${ }^{1,3}$
}

\begin{abstract}
Background: One of the consequences of the institutionalization of the elderly is the increased risk of falls. Games of video games have shown ability to improve balance in the elderly population, but there is no data on their use in institutionalized elderly. Objective: To evaluate the effects of training with Nintendo Wii on static balance and functional mobility in elderly living in Long-Term Care Facilities for Elderly. Methods: Participated in the research 10 institutionalized elderly, who underwent training with Nintendo Wii for eight weeks, twice a week. Functional mobility was assessed by the Timed Up and Go (TUG) test and the static balance by a force platform. Results: Significant improvement in TUG was observed ( $17.65 \pm 4.38$ s pre-treatment and $15.30 \pm 3.70$ s $p=0.03$ ). There was no significant improvement in the static equilibrium variables, but there was a tendency for improvement. Conclusion: The results demonstrated that the intervention with Nintendo Wii significantly improved the functional mobility, but with no improvement in the static balance in the institutionalized elderly.
\end{abstract}

Keywords: Postural Balance; Experimental Games; Aging.

\section{INTRODUCTION}

Currently in Brazil there are approximately 11 million people over 60 years old, and projections indicate that we will be the sixth country in the world in the number of the elderly in the year 2020, with approximately 32 million people in this age group ${ }^{(1)}$.

The aging process is accompanied by organic changes that may, alone or together, interfere with the health of the elderly. One of the important issues related to aging is the decrease in postural balance, with a more pronounced decline from the 60 years old ${ }^{(2)}$. In addition to the amplitude and frequency of body oscillation being greater in the elderly, correction of body stability is slower when compared to young people ${ }^{(2)}$. With advancing age, the planning and execution of coordinated movements is slowed down ${ }^{(2)}$. These changes increase the risk of falls, which, in addition to being responsible for the reduction of activities of daily living (ADLs), may lead to the institutionalization of the elderly ${ }^{(3,4)}$.

The institutionalized elderly population presents a profile of social isolation, inactivity and fragility, which presupposes the decline of physical capacities, besides the deterioration of the natural physiological capacities inherent to the aging process itself ${ }^{(5)}$. According to the World Health Organization $(2015)^{(6)}$, residents of Long-Term Care Institutions for Elders are considered to be a complex population due to factors associated with morbidity, polypharmacy and the high prevalence of dependence in the activities of daily living (ADLs). It is estimated that between $50 \%$ and $66 \%$ of the institutionalized elderly suffer at least one fall per year ${ }^{(7)}$, demonstrating change in postural balance.

Video games have become an effective tool coupled with rehabilitation because it is a portable and accessible device, easily adapting to the limitations of the elderly, and can be used to improve daily activity levels and increase physical fitness $^{(8,9)}$. Studies have shown that the Nintendo Wii can improve the balance of the elderly, since the games promote physical, auditory, proprioceptive and visual stimuli, besides being a motivating tool ${ }^{(8,10)}$. The Nintendo Wii with its games and training programs offers a rich sensory environment, associated with visual, auditory and proprioceptive feedback, also being able to improve attention and concentration in the movements of the game due to motor, oculomotor, cerebellar and limbic stimuli(11-13).

\footnotetext{
Corresponding author: Marcos Eduardo Scheicher. Adress: Departamento de Fisioterapia e Terapia Ocupacional, Faculdade de Filosofia e Ciências, Universidade Estadual Paulista (UNESP), Av. Hygino Muzzi Filho, 737. Bairro: Mirante, Marília (SP), Brazil. CEP: 17.525-000

Phone: +55 14 34149430. E-mail: marcos.scheicher@unesp.br

1 Instituto de Biociências, Universidade Estadual Paulista (UNESP), Rio Claro (SP), Brasil

${ }^{3}$ Departamento de Fisioterapia e Terapia Ocupacional, Faculdade de Filosofia e Ciências, Universidade Estadual Paulista (UNESP), Marília (SP), Brasil

Full list of author information is available at the end of the article.
}

Financial support: The authors declare that there was no financial support.

Submission date 14 March 2018; Acceptance date 03 July 2018; Publication date 15 October 2018 
Considering that institutionalized elderly have a great alteration of the postural balance, that video games can help in the improvement of the balance and the researches of training with Nintendo Wii, it is important to carry out researches with this tool in this population.

In view of the above, the objective of this study was to verify the effectiveness of the training with Nintendo Wii in static balance and mobility of institutionalized elderly.

\section{METHODS}

The present longitudinal analytic study was approved by the Research Ethics Committee of the Faculty of Philosophy and Sciences, UNESP/Marília, protocol number: 59664916.6.0000.5406. It was also approved by the Registro Brasileiro de Ensaios Clínicos (ReBEC), protocol number: RBR-9zbk7y.

The research was carried out in two LTC in the city of Marília-SP. The inclusion criteria were: elderly over 60 years old, with independent gait capacity and without the use of walking aid devices; who presented a score considered normal by the Mini Mental State Examination (MMSE), according to schooling, with the following cut marks: 20 for illiterates, 25 for 1 to 4 years of study, 26.5 for 5 to 8 years,
28 for 9 to 11 years and 29 for high levels of education ${ }^{(14)}$. The exclusion criteria were: elderly who presented sequelae of neurological and/or osteoarticular pathologies, uncorrected visual problems, postural hypotension and use of drugs interfering in postural balance. From 68 elderly living in the two LTC in the city of Marília/SP, 17 were included, because they were able to participate due to the eligibility criteria. From these, 10 started and finished the proposed training (Figure 1), forming a single group and the data analyzed were pre- and post-intervention (intention to treat).

The center of pressure (COP) data were obtained by a $\mathrm{AMTI}^{\circledR}$ (AccuSway Dual Top, Watertown, MA, EUA) force platform with a sampling frequency of $100 \mathrm{~Hz}$. Subjects were positioned at a distance of 2 meters from a fixed point placed 1.6 meters high, and were instructed to stare at the point during the test with the feet position standardized. The participants remained with their arms relaxed at the side of the trunk and barefoot, and were instructed to maintain a static posture as quiet as possible. Three evaluations were performed, with $30 \mathrm{~s}$ duration and one-minute interval between each evaluation, in the following situations: 1) closed base/open eyes; 2) closed base /closed eyes.

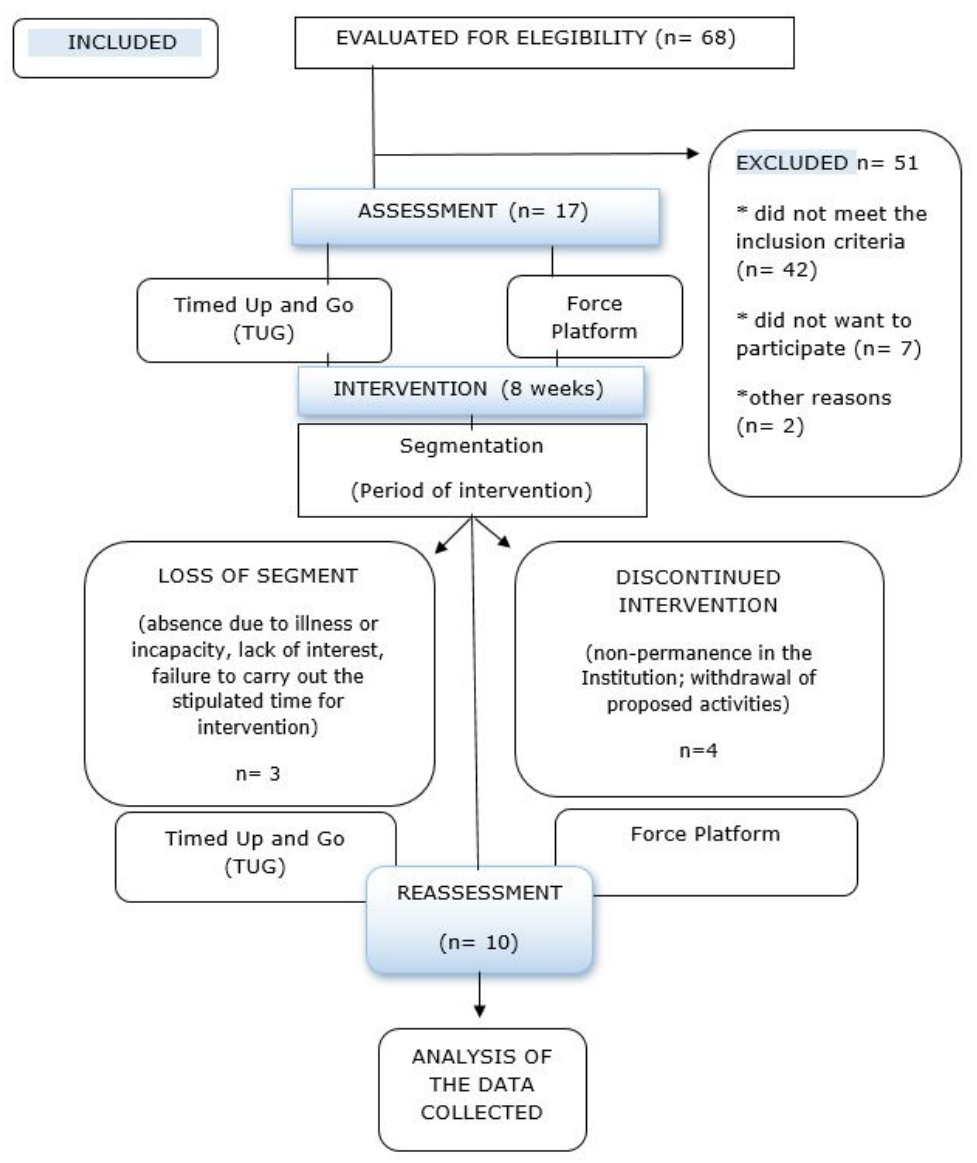

Figure 1. Flowchart of the study. 
The variables evaluated were: total displacement, anteroposterior displacement, medial-lateral displacement, COP mean velocity and area $95 \%$ of the ellipse.

The Timed Up and Go (TUG) test was used to evaluate participants' mobility, measuring in seconds the time spent by the volunteer to get up from a chair without the help of arms, walk a distance of 3 meters, turn around and return to the starting point. At the beginning of the test, the volunteer remains with his/her back resting on the back of the chair and, at the end, should return to the same position. The participant receives the instruction to "go" to perform the test and the time must be timed from the command voice until the moment the volunteer rests his back again on the chair. The test should be performed once for familiarization and a second time for timekeeping ${ }^{(15-17)}$. TUG is widely recommended as a predictor of risk of falls in the elderly, as it identifies balance and gait deficits. Therefore, lower values indicate better functional mobility, better postural balance, higher walking speed and, therefore, lower risk of falls ${ }^{(18)}$. According to Alexandre et al (2012), 12,4s is indicated time for Brazilian elderly ${ }^{(19)}$.

After the initial assessments in the force platform and TUG, the elderly spent a period of one week to familiarize themselves with the games proposed for the intervention, and that week was not included in the intervention period. In the period of familiarization, no facilitation was implemented to the participants, and the simulations were similar to the intervention protocol. The balance training was performed in 30-minute sessions, twice a week, for eight weeks ${ }^{(20-22)}$, with the use of Nintendo's Wii Fit ${ }^{\circledR}$ program, associated with the Wii Balance Board ${ }^{\circledR}$, platform that is sensitive to movement ${ }^{(23)}$. The games used for postural balance training were: Penguin Slide, Table Tilt and Tightrope. The level of difficulty of the games was provided by the virtual device itself, so that when the player reached his goal, a more advanced level of difficulty was unlocked. In the Penguin Slide game, the volunteer should be balanced on a block of ice while collecting fish, moving his body from side to side (Figure 2A). The Table Tilt game consists of a simulator of a platform with holes, in which the participant performed body oscillations on the platform in order to fit balls into the holes (Figure 2B). In the Tightrope game, the participant should walk and balance in a tightrope simulation, aiming to reach the end of the rope (Figure $2 \mathrm{C}$ ).
The normality of the data was made by the Shapiro-Wilk test. Pre- and post-treatment comparisons were made using the Wilcoxon test, with the level of significance of $p \leq 0.05$.

\section{RESULTS}

Pre and post-intervention force platform data can be visualized in Tables 1 and 2. It can be observed that there was no significant improvement in the variables evaluated with the

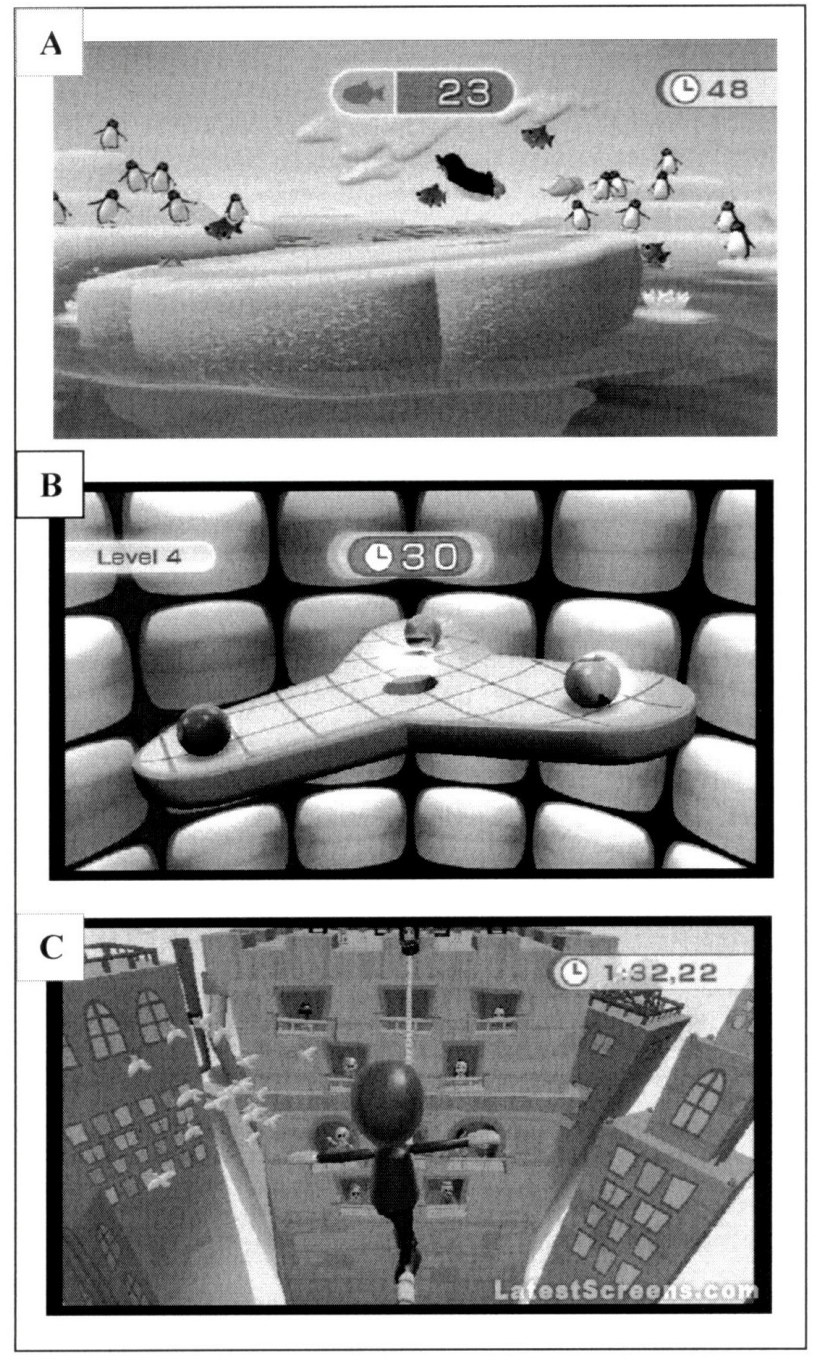

Figure 2. Games used in the balance training. A: Penguin Slide; B: Table Tilt; C: Tightrope. Source: internet.

Table 1. Static Balance in Pre and Post Intervention - Open Eyes.

\begin{tabular}{lccc}
\hline & Pre-treatment & Post-treatment & P \\
\hline Total displacement $(\mathrm{cm})$ & $67.65 \pm 21.85$ & $64.50 \pm 17.88$ & 0.37 \\
Total a-p displacement $(\mathrm{cm})$ & $1.12 \pm 0.42$ & $0.87 \pm 0.49$ & 0.19 \\
Total m-I displacement m-I (cm) & $4.59 \pm 1.80$ & $6.19 \pm 2.63$ & 0.23 \\
COP mean velocity $(\mathrm{cm} / \mathrm{s})$ & $2.27 \pm 0.72$ & $2.14 \pm 0.59$ & 0.32 \\
Area 95\% of ellipse $\left(\mathrm{cm}^{2}\right)$ & $6.12 \pm 2.7$ & $6.55 \pm 2.8$ & 0.32 \\
\hline
\end{tabular}

Note: a-p: anteroposterior; $\mathrm{m}$-I: mediolateral; COP: center of pressure. Data in mean \pm standard deviation. 
Table 2. Static Balance in Pre and Post Intervention - Closed Eyes.

\begin{tabular}{lccc}
\hline & Pre-treatment & Post-treatment & p \\
\hline Total displacement $(\mathrm{cm})$ & $84.77 \pm 26.19$ & $77.44 \pm 4.55$ & 0.27 \\
Total a-p displacement $(\mathrm{cm})$ & $0.94 \pm 0.56$ & $1.24 \pm 1.07$ & 0.84 \\
Total m-I displacement m-l (cm) & $4.32 \pm 1.69$ & $5.55 \pm 2.19$ & 0.08 \\
COP mean velocity $(\mathrm{cm} / \mathrm{s})$ & $2.81 \pm 0.87$ & $2.55 \pm 0.32$ & 0.23 \\
Area 95\% of ellipse $\left(\mathrm{cm}^{2}\right)$ & $6.56 \pm 3.21$ & $6.49 \pm 2.75$ & 0.92 \\
\hline
\end{tabular}

Note: a-p: anteroposterior; m-I: mediolateral; COP: center of pressure. Data in mean \pm standard deviation

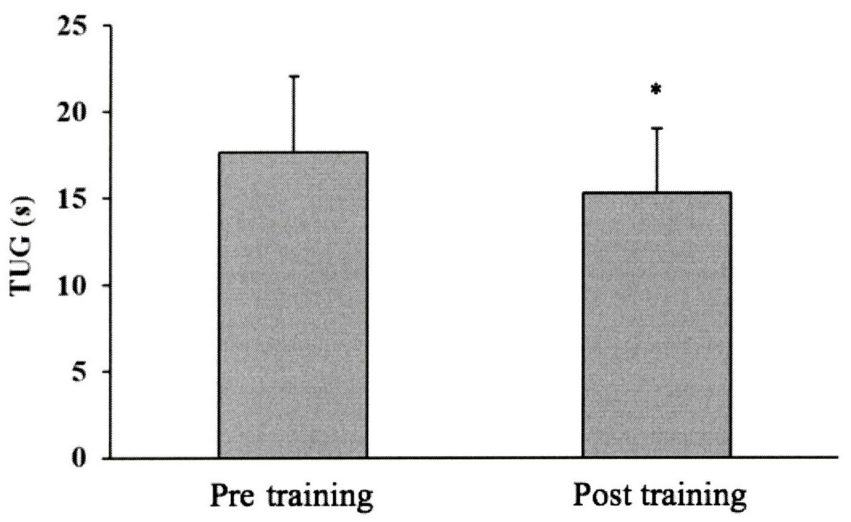

Figure 3. Mean time of the Timed Up and Go (TUG) test in institutionalized elderly $(n=10)$, pre $(17.65 \pm 4.38 \mathrm{~s})$ and post training $(15.30 \pm 3.70 \mathrm{~s})$ with Nintendo Wii. ${ }^{*} \mathrm{p}=0.03 . \Delta=2.35 \mathrm{~s}$.

proposed training. It is possible to observe, however, a trend of improvement in some variables.

The TUG values before and after the intervention are presented in Figure 3. It can be observed significant improvement of the values.

\section{DISCUSSION}

The present study aimed to evaluate the effects of training with Nintendo Wii on static balance and functional mobility in elderly living in Long-Term Care Institutions for the Elders.

The results showed that there was no significant pre and post-intervention difference in relation to the static balance. It is observed, however, that in some variables, there was a tendency for improvement.

In normal situations, in face of an imbalance, the sensorimotor biofeedback mechanism should respond quickly to prevent a possible fall by first activating the ankle joint and associated muscles and then the knee and hip joints ${ }^{(24)}$. With aging, there is a decrease in sensorimotor biofeedback and muscle strength, resulting in an inability of postural control by these mechanisms ${ }^{(25)}$.

It is suggested that the greater corporal oscillation of the elderly is due to the deterioration of the obtaining of accurate sensorial information and, as a consequence, the body oscillation would occur in greater magnitude with the purpose of the sensors to provide information about the oscillation and, thus, to trigger a muscular activity in order to reverse the body oscillation ${ }^{(26)}$. When the elderly can not adapt, the drastic fall situation can occur. The lower values of oscillation in some variables verified post-treatment indicate that, despite the non-statistical significance, there may have been a facilitation of the arrival of information to the CNS, triggering muscular activities that allowed the reduction of postural oscillation.

Regarding mobility (TUG), the data showed that there was a significant improvement with the proposed training $(p=0.03)$ and, although the elderly did not present an ideal time in the TUG post-intervention, according to the literature for reduction of the risk of falls, it is believed that through a larger training protocol (greater than 8 weeks) the elderly would reach the indicated value. The TUG involves common actions of daily life, fundamental for an independent life. It consists of complex activities for the elderly, as the one must have muscle strength of lower limbs and trunk to raise and sit without support, walk 3 meters, rotate $180^{\circ}$ and walk 3 meters again. These type of activities need power, agility and balance ${ }^{(27,28)}$. Therefore, lower values indicate better functional mobility, better balance, higher walking speed and, therefore, lower risk of falls, besides a possible greater independence in activities of daily living. The study of Verghese et al (2009) showed that every $0.1 \mathrm{~m} / \mathrm{s}$ of decreased in the gait speed there is a $7 \%$ increase in the risk of falling ${ }^{(29)}$. Based on this assumption, an increase in walking speed and, consequently, a decrease in TUG time, would suggest a decrease in the risk of falling.

The target audience for this research was the institutionalized elderly, whose physical, psychic and social behavior does not resemble elderly in the community ${ }^{(30-32)}$. According to the literature, the population living in LTC can be characterized by high dependence on the activities of daily living, multiple morbidities and polypharmacy ${ }^{(33,34)}$. The same study also mentions that these elderly spend about $75 \%$ of their time awake sitting or lying down, which contributes to intensify the decrease of mass and muscular strength. This suggests that this population may need more training time to achieve significant results in relation to static balance.

On the other hand, the training of balance with games has been studied as an additional tool to improve the postural 
balance. The study of Clark e Kraemer (2009) using games of video games in elderly with 89 years old demonstrated that the treatment was beneficial, providing significant improvement in functional mobility and representing a reduction in the risk of falls ${ }^{(35)}$.

Lange et al (2010) demonstrated in a study with hemiparetic subjects that the Nintendo Wii, besides favoring the static and dynamic balance, can also be able to produce constructive difficulties during the training, increasing the level and intensity of the game according to the individual capacity of each participant, generating ideal conditions of skills feedback and resulting in improvements in the functional abilities of the elderly ${ }^{(36)}$. Another factor that contributes to obtaining good results through the virtual platform is the fun and entertaining capacity that the interactive environment can provide the player, keeping the one motivated to the treatment ${ }^{(36)}$.

Miller et al (2012) and Menezes \& Bachion (2008) affirmed that in addition to improving functionality, the Nintendo Wii with its training protocols and the sensitivity of the Balance Board are able to generate postural reeducation, reducing the risk of falls and the fear of falling ${ }^{(37,38)}$.

There are few Brazilian and international studies in the literature that investigated the effect of virtual reality training on the balance of institutionalized elders. It was found 7 studies in the PubMed database and none in the Scielo database. Thus, this study becomes important, since it may initiate a new modality of treatment in long-term care institutions for the elders in Brazil.

\section{CONCLUSION}

There was no significant improvement in the static balance of institutionalized elderly undergoing training with Nintendo Wii. There was significant improvement in mobility after training with the Nintendo Wii games.

\section{AUTHOR'S CONTRIBUITIONS}

LAM: recruitment of participants, data collection and article development; LMF: recruitment of participants, data collection and article development; MES: orientation in data collection, statistical analysis and article development.

\section{CONFLICTS OF INTEREST}

The authors declare no conflict of interest

\section{AUTHORS DETAILS}

${ }^{2}$ Curso de Fisioterapia, Faculdade de Ensino Superior do Interior Paulista (FAIP), Marília (SP), Brasil

\section{REFERENCES}

1. IBGE. Projeção Da População Das Unidades Da Federação Por Sexo E Idade Para O Período 2000/2030. 2013; Available from: http://www.ibge.gov. br/home/estatistica/populacao/projecao_da_populacao/2013/

2. Bruniera CAV, Bento PCB, Canevari RO, Rogério FRPG, Rodacki ALF. Comparação da estabilidade postural em idosas residentes em instituição de longa permanência e praticantes de exercício físico. Rev da Educ Fis. 2014;25(2):223-30.
3. Perracini M. Prevenção e manejo de quedas. In: Ramos L, editor. Guia de geriatria e gerontologia. 2a . Barueri; 2005. p. 193-208.

4. Sousa JAV de, Stremel AIF, Grden CRB, Borges PK de O, Reche PM, Silva $\mathrm{JH}$ de $\mathrm{O}$ da. Risk of falls and associated factors in institutionalized elderly. Rev da Rede Enferm do Nord. 2016;17(3):416-21.

5. Preto L, Santos A, Mendes M, Novo A, Pimentel M. Deterioro funcional, miedo a caerse y composición corporal en ancianos institucionalizados. Enfermería Clínica. 2014;25(2):81-6.

6. World Health Organization. World report on ageing and health. 2015 [cited 2018 Mar 13]. Available from: http://apps.who.int/iris/ bitstream/10665/186463/1/9789240694811_eng.pdf

7. Rubenstein LZ, Josephson KR, Robbins AS. Falls in the Nursing Home. Ann Intern Med. 1994;121(6):442-51.

8. Dias RS, Sampaio ILA, Taddeo LS. Fisioterapia X Wii: a introdução do lúdico no processo de reabilitação de pacientes em tratamento fisioterápico. In: VIII Brazilian Symposium on Games and Digital Entertainment; 2009 Oct 8-10; Rio de Janeiro; 2009.

9. Gil-Gómez JA, Lloréns R, Alcñiz M, Colomer C. Effectiveness of a Wii balance board-based system (eBaViR) for balance rehabilitation: A pilot randomized clinical trial in patients with acquired brain injury. J Neuroeng Rehabil. 2011;8(1):30.

10. Williams MA, Soiza RL, Jenkinson AME, Stewart A. EXercising with C-omputers in L-ater L-ife (EXCELL) - Pilot and feasibility study of the acceptability of the Nintendo ${ }^{\circledR}$ Wiifit in community-dwelling fallers. BMC Res Notes. 2010;3.

11. Esculier JF, Vaudrin J, Bériault P, Gagnon K, Tremblay LE. Home-based balance training programme using Wii Fit with balance board for Parkinson's disease: A pilot study. J Rehabil Med. 2012;44(2):144-50.

12. Schenkman M, Butler RB. A Model for Multisystem Evaluation Treatment of Individuals with Parkinson's Disease. Phys Ther. 1989 Nov 1;69(11):932-43.

13. De la Fuente-Fernández R, Phillips AG, Zamburlini M, Sossi V, Calne DB, Ruth TJ, et al. Dopamine release in human ventral striatum and expectation of reward. Behav Brain Res. 2002;136(2):359-63.

14. Brucki SMD, Nitrin R, Caramelli P, Bertolucci PHF, Okamoto IH. Sugestões para o uso do mini-exame do estado mental no Brasil. Arq Neuropsiquiatr. 2003;61(3 B):777-81.

15. Podsiadlo D, Richadson S. The timed "Up and Go" test: A test of basic functional mobility for frail elderly persons. J Am Geriatr Soc. 1991;39(2):142-8.

16. Silva N de A, Menezes TN de. Capacidade funcional e sua associação com idade e sexo em uma população idosa. Rev Bras Cineantropometria e Desempenho Hum. 2014;16(3):359-70.

17. Ansai JH, Glisoi SF das N, Oliveira T de, Soares AT, Cabral K de N, Sera CTN, et al. Revisão de dois instrumentos clínicos de avaliação para predizer risco de quedas em idosos. Rev Bras Geriatr e Gerontol. 2014;17(1):177-89.

18. Tolson D, Rolland Y, Andrieu S, Aquino JP, Beard J, Benetos A, et al. International Association of Gerontology and Geriatrics: A Global Agenda for Clinical Research and Quality of Care in Nursing Homes. J Am Med Dir Assoc. 2011;12(3):184-9.

19. Alexandre TS, Meira DM, Rico NC, Mizuta SK. Accuracy of Timed Up and Go Test for screening risk of falls among community-dwelling elderly. Rev Bras Fisioter. 2012;16(5):381-8.

20. Dougherty J, Kancel A, Ramar C, Meacham C, Derrington S. The effects Of a multi-axis balance board intervention program in an elderly population. Mo Med. 2011;108(2):128-32.

21. Gatica Rojas V, Elgueta Cancino E, Vidal Silva C, Cantin López M, Fuentealba Arcos J. Impacto del Entrenamiento del Balance a través de Realidad Virtual en una Población de Adultos Mayores Impact of Balance Training with a Virtual Reality in Elderly. Int J Morphol. 2010;28(1):303-8.

22. Agmon M, Perry CK, Phelan E, Demiris G, Nguyen HQ. A pilot study of Wii Fit exergames to improve balance in older adults. J Geriatr Phys Ther. 2011;34(4):161-7. 
23. Clark RA, Bryant AL, Pua Y, McCrory P, Bennell K, Hunt M. Validity and reliability of the Nintendo Wii Balance Board for assessment of standing balance. Gait Posture. 2010;31(3):307-10.

24. Horlings CGC, Küng UM, van Engelen BGM, Voermans NC, Hengstman GJD, van der Kooi AJ, et al. Balance control in patients with distal versus proximal muscle weakness. Neuroscience. 2009;164(4):1876-86.

25. Mansfield A, Maki BE. Are age-related impairments in change-in-support balance reactions dependent on the method of balance perturbation? J Biomech. 2009;42(8):1023-31.

26. Barela J. Ciclo percepção-ação no desenvolvimento motor. In: Teixeira L, editor. Avanços em comportamento motor. São Paulo; 2001. p. 40-61.

27. Miotto JM, Chodzko-Zajko WJ, Reich JL, Supler MM. Reliability and validity of the fullerton functional fitness test: an independent replication study. J Aging Phys Activ. 1999;7(4):339-53.

28. Schenkman M, Hughes MA, Samsa G, Studenski S. The relative importance of strength and balance in chair rise by functionally impaired older individuals... presented, in part, at the Annual Meeting of Gerontological Society of America, Atlanta GA, 1994. J Am Geriatr Soc. 1996;1441-7.

29. Verghese J, Holtzer R, Lipton RB, Wang C. Quantitative gait markers and incident fall risk in older adults. Journals Gerontol - Ser A Biol Sci Med Sci. 2009;64(8):896-901.

30. Borges GF, Benedetti TRB, Farias SF. Atividade física habitual e capacidade funcional percebida de idosas do Sul do Brasil. Pensar a Prática. 2011 May 27;14(1):1-11.
31. Camarano AA, Kanso S, Fernandes D. Envelhecimento populacional, perda de capacidade laborativa e políticas públicas. Bol Merc Trab - Conjunt e Análise $n^{\circ}$ 54. 2013;21-9.

32. Ciolac E. Exercise training as a preventive tool for age-related disorders: a brief review. Clinics. 2013;68(5):710-7.

33. De Souto Barreto P, Lapeyre-Mestre M, Mathieu C, Piau C, Bouget C, Cayla $\mathrm{F}$, et al. A multicentric individually-tailored controlled trial of education and professional support to nursing home staff: Research protocol and baseline data of the IQUARE study. J Nutr Heal Aging. 2013;17(2):173-8.

34. de Souto Barreto P, Morley JE, Chodzko-Zajko W, H. Pitkala K, WeeningDjiksterhuis E, Rodriguez-Mañas L, et al. Recommendations on Physical Activity and Exercise for Older Adults Living in Long-Term Care Facilities: A Taskforce Report. J Am Med Dir Assoc. 2016;17(5):381-92.

35. Clark, R., Kraemer T. Clinical Use of Nintendo Wii ( TM ) Bowling Simulation to Decrease Fall Risk in an Elderly Resident: A Case Report. J Geriatr Phys Ther. 2009;32(4):174-80.

36. Lange B, Flynn S, Proffitt R, Chang C-Y, "Skip" Rizzo A. Development of an Interactive Game-Based Rehabilitation Tool for Dynamic Balance Training. Top Stroke Rehabil. 2010 Sep 5;17(5):345-52.

37. Miller CA, Hayes DM, Dye K, Johnson C, Meyers J. Using the nintendowii fit and bodyweight support to improve aerobic capacity, balance, gait ability, and fear of falling: Two case reports. J Geriatr Phys Ther. 2012;35(2):95-104.

38. Menezes RL de, Bachion MM. Estudo da presença de fatores de riscos intrínsecos para quedas, em idosos institucionalizados TT - Study of intrinsic risk factors for falls in institutionalized elderly people. Ciência \&amp; Saúde Coletiva. 2008;13(4):1209-18. 